IMSc/2017/06/04

\title{
Variety of $(d+1)$ dimensional
}

\section{Cosmological Evolutions with and without bounce in a class of LQC - inspired Models}

\author{
S. Kalyana Rama \\ Institute of Mathematical Sciences, HBNI, C. I. T. Campus, \\ Tharamani, CHENNAI 600 113, India. \\ email: krama@imsc.res.in
}

\begin{abstract}
The bouncing evolution of an universe in Loop Quantum Cosmolgy can be described very well by a set of effective equations, involving a function $\sin x$. Recently, we have generalised these effective equations to $(d+1)$ dimensions and to any function $f(x)$. Depending on $f(x)$ in these models inspired by Loop Quantum Cosmolgy, a variety of cosmological evolutions are possible, singular as well as non singular. In this paper, we study them in detail. Among other things, we find that the scale factor $a(t) \propto t^{\frac{2 q}{(2 q-1)(1+w) d}}$ for $f(x)=x^{q}$, and find explicit Kasner-type solutions if $w=2 q-1$ also. A result which we find particularly fascinating is that, for $f(x)=\sqrt{x}$, the evolution is non singular and the scale factor $a(t)$ grows exponentially at a rate set, not by a constant density, but by a quantum parameter related to the area quantum.
\end{abstract}




\section{Introduction}

Consider a homogeneous expanding universe whose constituents have the density $\rho$ and the pressure $p$ obeying the condition $\rho+p>0$. In Einstein's theory of general relativity, as one goes back in time, the size of such an universe decreases and vanishes at a finite time in the past. The curvature invariants then diverge, the density diverges, and the evolution of the universe becomes singular.

In a quantum theory of gravity, among other things, such singularities are expected to be resolved. In Loop Quantum Cosmology (LQC) [1] - [5] which arises in a $(3+1)$ dimensional quantum gravity theory based on Loop Quantum Gravity (LQG) [6, 7], such singularities are indeed resolved due to quantum effects. In LQC, as one goes back in time, the size of the universe decreases, reaches a non zero minimum, and increases to $\infty$ in the infinite past. The curvature invariants remain finite, the density remains bounded from above and, therefore, the evolution of the universe in LQC has a bounce and is non singular.

It turns out that the quantum dynamics of such a non singular evolution in LQC can be described very well by a set of effective equations $[3,4,8,9$, 10, 11]. These equations reduce to Einstein's equations in the classical limit. These effective equations, in our notation, involve a certain function $f(x)$, see equations (22) and (23) where it will be first introduced. The variable $x$ will turn out to be related to the time derivative of the scale factor, and the function $f$ will dictate the precise relation between them. In LQC, this function $f(x)=\sin x$ and in the classical limit which leads to Einstein's equations, $f(x)=x$. In fact, Einstein's equations follow upto a scaling of time whenever $f(x)$ vanishes linearly. Thus, in LQC where $f(x)=\sin x$, the evolution is same as in Einstein's theory in the limits $x \rightarrow 0$ as well as $x \rightarrow \pi$. These limits constitute the two ends of the bouncing evolution in LQC where the size of the universe evolves to infinity, and the evolution in these two asymptotic limits is same as in Einstein's theory.

In a recent paper [12], we have generalised the effective LQC equations. Our generalisations are empirical and not derived from any underlying theory. But they are simple, straightforward, and natural. We generalised from $(3+1)$ to $(d+1)$ dimensions where $d \geq 3,{ }^{1}$ and generalised the trigonometric

\footnotetext{
${ }^{1}$ There exists a $(d+1)$ dimensional LQG formulation, given in $[13,14,15]$. Our pre-
} 
function as well as a $\bar{\mu}$ function which appear in the effective LQC equations. These generalised equations describe the cosmological evolution of a $(d+1)$ dimensional homogeneous, anisotropic universe and may be considered as a class of LQC - inspired models.

In this paper, we will consider only the generalisation of the trigonometric function, keeping the $\bar{\mu}$ function similar to that in the so-called $\bar{\mu}$-scheme. In LQC, the function $f(x)=\sin x$ is determined by the underlying theory. In the present LQC - inspired models, the generalisation is empirical and no underlying theory is invoked which may determine $f(x)$. This feature is a shortcoming of the present models. But taking it as a strength, one may consider a variety of functions $f(x)$ from a completely general point of view, study the corresponding evolution, and gain insights into the various types of singular and non singular evolutions possible.

Here, we follow this approach and study a variety of $(d+1)$ dimensional cosmological evolutions in the LQC - inspired models, corresponding to a variety of possible behaviours of the function $f(x)$. Assuming that $p=w \rho$ where $w$ is a constant and $(1+w)>0$, we study both isotropic and anisotropic cases. The resulting evolutions may or may not have a bounce, and may be singular or non singular. In the isotropic case, for several functions $f(x)$, we also find the potential $V(\sigma)$ for a minimally coupled scalar field $\sigma$ which may give rise to the equation of state $p=w \rho$. See [18] for analogous potential $V(\sigma)$ in LQC.

In general, given a function $f(x)$, it is not possible to obtain explicit solutions to the relevant equations of motion. However, these equations have a shift and a scaling symmetry which may be used to understand several important features of the evolutions. In this paper, we first consider the cases with non trivial functions $f(x)$ where explicit solutions may be obtained. For the isotropic case, explicit solutions may be obtained for the functions $f(x)=\sin x, x^{q}$, and $e^{x}$. For the anisotropic case, explicit solutions may be obtained for the function $f(x)=x^{q}$ if $w=2 q-1$. These anisotropic solutions are the analogs of the standard Kasner-type solutions in Einstein's theory.

We then study a class of functions where $f(x) \rightarrow x$ as $x \rightarrow 0$ so that the evolution in this limit is same as in Einstein's theory. Thus, in this limit,

liminary analysis [16], see [17] also, suggests that one can derive the LQC analogs of the effective equations in $(d+1)$ dimensions, with $f(x)=\sin x$. 
the time $t \rightarrow \infty$, the scale factor $a \rightarrow \infty$, and the universe is expanding. As $x$ increases, $t$ decreases, $a$ decreases, and the universe decreases in size. Assuming that $f(x)$ remains positive and all its derivatives remain bounded for $0<x<x_{r}$, we study possible asymptotic behaviours in cases where $f(x) \rightarrow 0$ or $\infty$ in the limit $x \rightarrow x_{r}$, and $x_{r}$ itself may be finite or infinite. We study the cases where the function $f(x) \propto\left(x_{r}-x\right)^{q}$ in the limit $x \rightarrow x_{r}$ and also the cases where, in the limit $x \rightarrow \infty$, the function $f(x) \propto x^{q}$, or $f(x) \rightarrow$ (const) , or $f(x) \propto e^{-b x}$. Such asymptotic behaviours are quite natural and, hence, they may apply to a wide class of functions $f(x)$. Together with the shift and the scaling symmetries, the explcit solutions obtained earlier may now be used to describe the asymptotic evolutions in all of these cases.

The main results of our study are the following. The explicit solution presented in this paper for $f(x)=\sin x$ generalises the $(3+1)$ dimensional LQC solution given in [18] to $(d+1)$ dimensions. The explicit solutions presented in this paper for $f(x)=x^{q}$ generalise the evolution of the scale factor $a$ in Einstein's theory to $a(t) \propto t^{\frac{2 q}{(2 q-1)(1+w) d}}$. When $w=2 q-1$, they also generalise the standard Kasner-type solutions in Einstein's theory.

We find both singular and non singular evolutions. The asymptotic behaviours of the scale factor in the isotropic cases are tabulated in Tables I and II. Here, in the Introduction, we point out a few cases of non singular evolutions. In all these cases, in the limit $x \rightarrow 0$, the function $f(x) \rightarrow x$, the scale factor $a \rightarrow \infty$, the time $t \rightarrow \infty$, and $a$ and $t$ decrease as $x$ increases.

- For the case where $f(x) \propto\left(x_{r}-x\right)^{q}$ as $x \rightarrow x_{r}$ and $2 q \geq 1$, the evolution is non singular and has a bounce. In the limit $x \rightarrow x_{r}$, one has $t \rightarrow-\infty$ and $a \rightarrow \infty$. The function $f(x)=\sin x$ falls under this case and corresponds to $x_{r}=\pi$ and $q=1$.

- For the case where $f(x) \propto x^{q}$ as $x \rightarrow \infty$ and $2 q \leq 1$, the evolution is non singular. In the limit $x \rightarrow \infty$, one has $t \rightarrow-\infty$; and $a \rightarrow 0$ if $0<2 q \leq 1, a \rightarrow$ (const) if $q=0$, and $a \rightarrow \infty$ if $q<0$. The evolution for the $q=0$ case in this limit is similar to that expected in the Hagedorn phase of string/M theory [19] - [24].

We find that the case where $f(x)=x^{q}$ and $0<2 q<1$ exhibit an interesting feature: There is a singularity when the universe is increasing to 
infinite size, and no singularity when it is contracting to zero size. This is opposite to what one usually comes across in Einstein's theory.

We find that the $2 q=1$ case where $f(x)=\sqrt{x}$ is particularly fascinating. The evolution now is non singular and it straddles the border between the singular and non singular evolution : in the limit $x \rightarrow 0$, the evolution is non singular for $2 q>1$ and is singular for $2 q<1$; in the limit $x \rightarrow \infty$, the evolution is singular for $2 q>1$ and is non singular for $2 q<1$.

Also, for $2 q=1$, the scale factor $a$ grows exponentially and the exponential rate is set, not by a constant density, but by a quantum parameter which is related to the area quantum as in LQC. The density $\rho \propto a^{-(1+w) d}$ and is not constant. This exponential growth of the scale factor is, therefore, unlike that which occurs in Einstein's theory due to a positive cosmological constant for which $w=-1$ and the density is constant. We find these results intriguing and fascinating but their significance, if any, is not clear to us at present.

This paper is organised as follows. In Section 2, we present the equations of motion for a $(d+1)$ dimensional homogeneous anisotropic universe in Einstein's theory. In Section 3, we present the effective equations of motion in LQC. In Section 4, we present the generalised effective equations of motion for our LQC - inspired models. In Section 5, we describe the general features of these models. In Section 6, we present explicit isotropic solutions and use them to study other asymptotic evolutions. In Tables I and II, we have also tabulated the asymptotic behaviours of the scale factor in the isotropic cases so that the results may be seen at a glance. In Section 7, we present explicit anisotropic solutions and study other anisotropic asymptotic evolutions. In Section 8, we present a brief summary and conclude by mentioning several issues for further studies.

\section{2. $(d+1)$ dimensional Einstein's equations}

Let the spacetime be $(d+1)$ dimensional where $d \geq 3$ and let $x^{i}$, $i=1,2, \cdots, d$, denote the spatial coordinates. Also, let the $d$-dimensional space be toroidal and let $L_{i}$ denote the coordinate length of the $i^{\text {th }}$ direction. Consider a homogeneous and anisotropic universe whose line element $d s$ is 
given by

$$
d s^{2}=-d t^{2}+\sum_{i} a_{i}^{2}\left(d x^{i}\right)^{2}
$$

where the scale factors $a_{i}$ depend on $t$ only. Here and in the following, we will explicitly write the indices to be summed over since the convention of summing over repeated indices is not always applicable. Einstein's equations are given, in the standard notation with $\kappa^{2}=8 \pi G_{d+1}$, by

$$
R_{A B}-\frac{1}{2} g_{A B} R=\kappa^{2} T_{A B} \quad, \quad \sum_{A} \nabla^{A} T_{A B}=0
$$

where $A, B=(0, i)$ and $T_{A B}$ is the energy momentum tensor. We assume that $T_{A B}$ is diagonal and that its diagonal elements are given by

$$
T_{00}=\rho, \quad T_{i i}=p_{i}
$$

where $\rho$ is the density and $p_{i}$ is the pressure in the $i^{t h}$ direction. Defining the quantities $\lambda^{i}, \Lambda, a, G_{i j}$, and $G^{i j}$ by

$$
\begin{gathered}
e^{\lambda^{i}}=a_{i}, \quad e^{\Lambda}=\prod_{i} a_{i}=a^{d} \quad \longrightarrow \quad \Lambda=\sum_{i} \lambda^{i}, \\
G_{i j}=1-\delta_{i j}, \quad \sum_{i} G^{i j} G_{j k}=\delta_{k}^{i} \quad \longrightarrow \quad G^{i j}=\frac{1}{d-1}-\delta^{i j},
\end{gathered}
$$

and after a straightforward algebra, Einstein's equations (2) give

$$
\begin{aligned}
\sum_{i j} G_{i j} \lambda_{t}^{i} \lambda_{t}^{j} & =2 \kappa^{2} \rho \\
\lambda_{t t}^{i}+\Lambda_{t} \lambda_{t}^{i} & =\kappa^{2} \sum_{j} G^{i j}\left(\rho-p_{j}\right) \\
\rho_{t}+\sum_{i}\left(\rho+p_{i}\right) \lambda_{t}^{i} & =0
\end{aligned}
$$

where the $t$-subscripts denote derivatives with respect to $t$. It follows from equations (5) that

$$
\left(\lambda_{t}^{i}-\lambda_{t}^{j}\right)_{t}+\Lambda_{t}\left(\lambda_{t}^{i}-\lambda_{t}^{j}\right)=\kappa^{2}\left(p_{i}-p_{j}\right) .
$$


If the pressures are isotropic then $p_{i}=p$ for all $i$, and we get

$$
\lambda_{t}^{i}-\lambda_{t}^{j}=(\text { const }) e^{-\Lambda}
$$

and

$$
\rho_{t}+\Lambda_{t}(\rho+p)=0
$$

If the scale factors are also isotropic then $a_{i}=a$ and $\lambda_{t}^{i}=\frac{a_{t}}{a} \equiv H$ for all $i$, and equations (4) and (5) give

$$
\begin{aligned}
H^{2} & =\frac{2 \kappa^{2} \rho}{d(d-1)} \\
H_{t} & =-\frac{\kappa^{2}(\rho+p)}{d-1} .
\end{aligned}
$$

Note that if $p_{i}=w_{i} \rho$ where $w_{i}$ are constants then equations (4) - (6) can be solved exactly [12]. Also note that for a minimally coupled scalar field $\sigma$, which has a potential $V(\sigma)$ and depends on $t$ only, it is a standard result that the density $\rho$ and the pressure $p$ are given by

$$
\rho=\frac{\left(\sigma_{t}\right)^{2}}{2}+V \quad, \quad p_{i}=p=\frac{\left(\sigma_{t}\right)^{2}}{2}-V .
$$

The equation of motion for the field $\sigma$ is given by

$$
\sigma_{t t}+\Lambda_{t} \sigma_{t}+\frac{d V}{d \sigma}=0
$$

which also follows from equations (9) and (12). Furthermore, in the isotropic case, one can construct a potential $V$ for the scalar field $\sigma$ such that $p=w \rho$ where $w$ is a constant: Writing $\sigma_{t}^{2}=(1+w) \rho$ and $2 V=(1-w) \rho$ and after some manipulations involving equations (10) and (11), see comments below equation (41), it can be shown that the required potential is given by

$$
V(\sigma) \propto(1-w) e^{-2 c_{w} \sigma}, \quad c_{w}=\sqrt{\frac{(1+w) \kappa^{2} d}{2(d-1)}} .
$$




\section{3. $(3+1)$ dimensional Effective LQC equations}

In this section, we mention briefly the main steps involved in obtaining the effective equations of motion in the $(3+1)$ dimensional Loop Quantum Cosmology (LQC). A detailed derivation and a complete description of various terms and concepts mentioned below are given in the review [4]. Below, we present the LQC expressions in a form which can be readily generalised.

Let the three dimensional space be toroidal and let the line element $d s$ be given by equation (1) where $d=3$ now, and $i=1,2,3$. Let $L_{i}$ and $a_{i} L_{i}$ be the coordinate and the physical lengths of the $i^{\text {th }}$ direction. In the Loop Quantum Gravity (LQG) formalism, the canonical pairs of phase space variables consist of an $S U(2)$ connection $A_{a}^{i}=\Gamma_{a}^{i}+\gamma K_{a}^{i}$ and a triad $E_{i}^{a}$ of density weight one. Here $\Gamma_{a}^{i}$ is the spin connection defined by the triad $e_{i}^{a}, \quad K_{a}^{i}$ is related to the extrinsic curvature, and $\gamma>0$ and $\approx 0.2375$ is the Barbero - Immirzi parameter of LQG, its numerical value being suggested by the black hole entropy calculations. For the anisotropic universe, whose line element $d s$ is given in equation (1) with $d=3$, one has $A_{a}^{i} \propto \hat{c}_{i}$ and $E_{i}^{a} \propto \hat{p}_{i}$ where $\hat{c}_{i}$ will turn out to be related to the time derivative of $a_{i}$, and $\hat{p}_{i}$ is given by

$$
\hat{p}_{i}=\frac{V}{a_{i} L_{i}}, \quad V=\prod_{j} a_{j} L_{j}
$$

with $V$ being the physical volume. The full expressions for $A_{a}^{i}$ and $E_{i}^{a}$ contain various fiducial triads, cotriads, and other elements, and are given in $[4,9]$. The non vanishing Poisson brackets among $\hat{c}_{i}$ and $\hat{p}_{j}$ are given by

$$
\left\{\hat{c}_{i}, \hat{p}_{j}\right\}=\gamma \kappa^{2} \delta_{i j}
$$

where $\kappa^{2}=8 \pi G_{4}$. The effective equations of motion are given by the 'Hamiltonian constraint' $\mathcal{C}_{H}=0$ and by the Poisson brackets of $\hat{p}_{i}$ and $\hat{c}_{i}$ with $\mathcal{C}_{H}$ which give the time evolutions of $\hat{c}_{i}$ and $\hat{p}_{i}$ : namely, by

$$
\mathcal{C}_{H}=0, \quad\left(\hat{p}_{i}\right)_{t}=\left\{\hat{p}_{i}, \mathcal{C}_{H}\right\}, \quad\left(\hat{c}_{i}\right)_{t}=\left\{\hat{c}_{i}, \mathcal{C}_{H}\right\} .
$$

Given that Einstein's action for gravity is known, it is to be expected that there exists a classical $\mathcal{C}_{H}$, the Poisson brackets with which lead to the classical dynamics given by Einstein's equations. Non trivially, and as reviewed in detail in [4], there also exists an effective $\mathcal{C}_{H}$, the Poisson brackets 
with which lead to the equations of motion which describe very well the quantum dynamics of LQC. The effective $\mathcal{C}_{H}$ reduces to the classical one in a suitable limit.

The expression for the $\mathcal{C}_{H}$ is of the form

$$
\mathcal{C}_{H}=H_{\text {grav }}\left(\hat{p}_{i}, \hat{c}_{i}\right)+H_{\text {mat }}\left(\hat{p}_{i} ;\left\{\phi_{\text {mat }}\right\},\left\{\pi_{\text {mat }}\right\}\right)
$$

where $H_{\text {grav }}$ denotes the effective gravitational Hamiltonian and $H_{\text {mat }}$ denotes a generalised matter Hamiltonian. In the matter sector, the density $\rho$ and the pressure $p_{i}$ in the $i^{t h}$ direction are defined by

$$
\rho=\frac{H_{m a t}}{V}, \quad p_{i}=-\frac{a_{i} L_{i}}{V} \frac{\partial H_{m a t}}{\partial\left(a_{i} L_{i}\right)} .
$$

The pressure $p_{i}$ is thus, as to be physically expected, proportional to the change in energy per fractional change in the physical length in the $i^{\text {th }}$ direction. As indicated in equation (18), $H_{m a t}$ is assumed to be independent of $\hat{c}_{i}$. Since $\hat{c}_{i}$ will turn out to be related to $\left(a_{i}\right)_{t}$, this assumption is equivalent to assuming that matter fields couple to the metric fields but not to the curvatures. This assumption can also be shown to lead to the conservation equation (6), namely to

$$
\rho_{t}=\left(\frac{H_{m a t}}{V}\right)_{t}=-\sum_{i}\left(\rho+p_{i}\right) \lambda_{t}^{i},
$$

irrespective of what $H_{\text {grav }}$ is [12].

In the gravitational sector, the effective $H_{\text {grav }}$, from which the LQC dynamics follow, is given in the so-called $\bar{\mu}$-scheme by

$$
H_{\text {grav }}=-\frac{V}{\gamma^{2} \lambda_{q m}^{2} \kappa^{2}}\left(\sin \left(\bar{\mu}^{1} \hat{c}_{1}\right) \sin \left(\bar{\mu}^{2} \hat{c}_{2}\right)+\text { cyclic terms }\right)
$$

where $V=\sqrt{\hat{p}_{1} \hat{p}_{2} \hat{p}_{3}}$ is the physical volume, $\lambda_{q m}^{2}=\sqrt{\frac{3}{4}} \gamma \kappa^{2}$ is the quantum of area, and $\bar{\mu}^{i}=\frac{\lambda_{q m} \hat{p}_{i}}{V}$ in the $\bar{\mu}$-scheme. Classical $H_{\text {grav }}$ follows in the limit $\bar{\mu}^{i} \hat{c}_{i} \rightarrow 0$ where $\sin \left(\bar{\mu}^{i} \hat{c}_{i}\right) \rightarrow \bar{\mu}^{i} \hat{c}_{i}$. 


\section{4. $(d+1)$ dimensional LQC - inspired models}

In a recent paper [12], we generalised the effective LQC equations. Our generalisations are empirical and not derived from any underlying theory. But they are simple, straightforward, and natural. And, they may be used to model a variety of non singular cosmological evolutions. In [12], we generalised from $(3+1)$ to $(d+1)$ dimensions where $d \geq 3$, and generalised the trigonometric and the $\bar{\mu}$ functions appearing in the effective $H_{\text {grav }}$ in equation (21). In this paper, we will consider only the generalisation of the trigonometric function, keeping the $\bar{\mu}$ function as in the $\bar{\mu}$-scheme.

We now present the generalised effective equations of our LQC - inspired models, expressing them so that they resemble equations (4) and (5) as closely as possible. For the purpose of this generalisation, we have already presented the LQC expressions in a form which can be readily taken over. Upon generalisation, we have the following.

- The index $i=1,2, \cdots, d$ now in the LQC expressions.

- The canonical pairs of phase space variables are given by $\hat{c}_{i}$ which will be related to $\left(a_{i}\right)_{t}$, and $\hat{p}_{i}$ which is given by equation (15). The non vanishing Poisson brackets among $\hat{c}_{i}$ and $\hat{p}_{j}$ are given by equation (16) where now $\kappa^{2}=8 \pi G_{d+1}$ and $\gamma$ may characterise the quantum of the $(d-1)$ dimensional area given by $\lambda_{q m}^{d-1} \sim \gamma \kappa^{2}[13,14,15,25]$.

- The effective equations of motion are given by equation (17) where $\mathcal{C}_{H}$ is of the form given in equation (18). In the matter sector, the density $\rho$ and the pressures $p_{i}$ are given by equations (19), and they satisfy the standard conservation equation (20).

- In the gravitational sector, the effective $H_{\text {grav }}$ in equation (21) is now generalised to

$$
H_{\text {grav }}=-\frac{V \mathcal{G}}{\gamma^{2} \lambda_{q m}^{2} \kappa^{2}}, \quad \mathcal{G}=\frac{1}{2} \sum_{i j} G_{i j} f^{i} f^{j}=\sum_{i j(i<j)} f^{i} f^{j}
$$

where $V=\left(\prod_{i} \hat{p}_{i}\right)^{\frac{1}{d-1}}$ is the $d$ dimensional physical volume and

$$
f^{i}=f\left(m^{i}\right), \quad m^{i}=\bar{\mu}^{i} \hat{c}_{i}, \quad \bar{\mu}^{i}=\frac{\lambda_{q m} \hat{p}_{i}}{V} .
$$


The function $f(x)$ which appears in equation (23) is arbitrary, but with the only requirement that $f(x) \rightarrow x$ as $x \rightarrow 0$ so that classical $H_{\text {grav }}$ is obtained in the limit $m^{i} \rightarrow 0$. Clearly, LQC is obtained upon setting $d=3$ and $f(x)=\sin x$.

\section{Equations of motion}

Equations of motion can now be obtained using the generalised $H_{\text {grav }}$ given in equation $(22)$. They will describe the evolution of a $(d+1)$ dimensional homogeneous anisotropic universe in our LQC - inspired models. The required algebra is straightforward but involved, see [12] for details. In this paper, we present only the final equations which suffice for our purposes here. The resulting equations of motion, expressed so that they resemble equations (4) and (5) as closely as possible, are given by

$$
\begin{aligned}
\sum_{i j} G_{i j} f^{i} f^{j} & =2 \gamma^{2} \lambda_{q m}^{2} \kappa^{2} \rho \\
\left(m^{i}\right)_{t}+\sum_{j} \frac{\left(m^{i}-m^{j}\right) X_{j}}{(d-1) \gamma \lambda_{q m}} & =-\gamma \lambda_{q m} \kappa^{2} \sum_{j} G^{i j}\left(\rho+p_{j}\right) \\
\left(\gamma \lambda_{q m}\right) \lambda_{t}^{i} & =\sum_{j} G^{i j} X_{j}
\end{aligned}
$$

where we have defined

$$
X_{i}=g_{i} \sum_{j} G_{i j} f^{j}, \quad g_{i}=\frac{d f\left(m^{i}\right)}{d m^{i}} .
$$

Equations (24) - (26) give the conservation equation (6). Also, equation (26) gives $(d-1)\left(\gamma \lambda_{q m}\right) \Lambda_{t}=\sum_{j} X_{j}$, and equation (25) then gives

$$
\left(m^{i}-m^{j}\right)_{t}+\Lambda_{t}\left(m^{i}-m^{j}\right)=\gamma \lambda_{q m} \kappa^{2}\left(p_{i}-p_{j}\right) .
$$

If the pressures are isotropic then $p_{i}=p$ for all $i$, and we get

$$
m^{i}-m^{j}=\mu^{i j} e^{-\Lambda}
$$


where $\mu^{i j}$ are constants. In the completely isotropic case, we have

$$
\left(p_{i}, m^{i}, f^{i}, a_{i}\right)=(p, m, f, a)
$$

and, hence,

$$
\lambda_{t}^{i}=\frac{a_{t}}{a}=H \quad, \quad X_{i}=(d-1) g f \quad, \quad g_{i}=g=\frac{d f}{d m} .
$$

Equations $(24)-(26)$ then give

$$
\begin{aligned}
f^{2} & =\frac{2 \gamma^{2} \lambda_{q m}^{2} \kappa^{2} \rho}{d(d-1)}=\frac{\rho}{\rho_{q m}} \\
m_{t} & =-\frac{\gamma \lambda_{q m} \kappa^{2}}{d-1}(\rho+p) \\
H & =\frac{g f}{\gamma \lambda_{q m}}
\end{aligned}
$$

where $\rho_{q m}=\frac{d(d-1)}{2 \gamma^{2} \lambda_{q m}^{2} \kappa^{2}}$. Equations (31) - (33) give the conservation equation (9). We will assume that $\rho+p>0$. Then equation (32) gives $m_{t}<0$. Hence, $m$ will increase monotonically as $t$ decreases. Also, equations (31) and (33) give

$$
H^{2}=\frac{2 \kappa^{2}\left(\rho g^{2}\right)}{d(d-1)}
$$

If $g$ can be expressed in terms of $f$ then, using $f^{2}=\frac{\rho}{\rho_{q m}}$, one can express $H^{2}$ in terms of $\rho$ alone. For example,

$$
f(x)=\sin x \quad \Longrightarrow \quad H^{2} \propto \rho\left(1-\frac{\rho}{\rho_{q m}}\right)
$$

and $f(x)=f(x ; n)$ where $n$ is a positive integer and

$$
f(x ; n)=1-\left(1-\frac{x}{x_{*}}\right)^{2 n} \Longrightarrow H^{2} \propto \rho\left(1-\sqrt{\frac{\rho}{\rho_{q m}}}\right)^{\frac{2 n-1}{n}} .
$$

Note that $f(x ; n)$ is a class of functions parametrised by $n$, that $n$ indicates the flatness of $f$ near its maximum at $x_{*}$, and that $f(x ; n) \rightarrow \frac{2 n}{x_{*}}\left(2 x_{*}-x\right)$ in the limit $x \rightarrow 2 x_{*}$. 


\section{General Features in the LQC - inspired models}

Obtaining the cosmological evolution of the universe in the LQC - inspired models further requires specifying the equations of state which give the pressures $p_{i}$ in terms of $\rho$. Once the equations of state are given, or assumed, equations (24) - (26) can be solved for a given set of initial values $\left(m_{0}^{i}, \lambda_{0}^{i}\right)$ at $t=t_{0}$. Here and in the following, the 0 -subscripts will denote the initial values at some initial time $t_{0}$. Given $m_{0}^{i}$, the values $\left(f_{0}^{i}, g_{i 0}, X_{i 0}\right)$ follow. Equation (24) then gives $\rho_{0}$; equations of state give $p_{i 0}$; and, equation (25) gives $\left(m^{i}\right)_{t 0}$ from which the value of $m^{i}$ at $t_{0} \pm \delta t$ follows. Repeating this procedure will give $m^{i}$ and $\left(f^{i}, g_{i}, X_{i}\right)$ for all $t$. The initial value $\lambda_{0}^{i}$ and equation (26) for $\lambda_{t}^{i}$ then determine $\lambda^{i}$ for all $t$. Thus, equations $(24)-(26)$ can always be solved numerically.

However, in general, it is not possible to obtain analytical solutions explicitly. Nevertheless, it is possible to understand several features of the evolution, as we now describe in a series of remarks.

Remark (1) : Let $f\left(m^{i}\right)=m^{i}$. Then $g_{i}=1$, and equation (26) and the definition of $m^{i}$ give

$$
\gamma \lambda_{\text {qm }} \lambda_{t}^{i}=m^{i}, \quad \hat{c}_{i}=\gamma L_{i}\left(a_{i}\right)_{t} .
$$

This shows that $\hat{c}_{i}$ is related to $\left(a_{i}\right)_{t}$. After a little algebra, equations (24) and (25) give equations (4) and (5), the Einstein's equations for a $(d+1)$ dimensional homogeneous anisotropic universe.

Remark (2) : Equations (24) - (26) remain invariant under the shift $m^{i} \rightarrow \tilde{m}^{i}=m^{i}+m_{s}$ where $m_{s}$ is constant and same for all $i$. Hence, $f\left(m^{i}\right)$ and $f\left(\tilde{m}^{i}\right)$ will lead to the same evolution.

Remark (3): Under the scaling $m^{i} \rightarrow \tilde{m}^{i}=\alpha m^{i}$ and $t \rightarrow \tilde{t}=\alpha t$ where $\alpha$ is constant and same for all $i$, and $\rho$ and $p_{i}$ remain unchanged, we have

$$
\lambda_{t}^{i} \rightarrow \lambda_{\tilde{t}}^{i}=\frac{\lambda_{t}^{i}}{\alpha}, \quad \rho_{t} \rightarrow \rho_{\tilde{t}}=\frac{\rho_{t}}{\alpha},
$$

and, from equations (27),

$$
g_{i} \rightarrow \tilde{g}_{i}=\frac{d f\left(\tilde{m}^{i}\right)}{d \tilde{m}^{i}}=\frac{g_{i}}{\alpha}, \quad X_{i} \rightarrow \tilde{X}_{i}=\frac{X_{i}}{\alpha} .
$$


It then follows that equations (6) and (24) - (26) remain invariant under these scalings. The invariances under the shift and the scaling then imply that $\left\{f\left(m^{i}\right), t\right\}$ and $\left\{f\left(\alpha m^{i}+m_{s}\right)\right.$, at $\}$ will lead to the same evolution.

Note that these invariance properties are accidental, are not present even for the more general models presented in [12], and arise from the structure of the equations (24) - (26) considered here. Nevertheless, these properties are useful practically. The scaling with $\alpha=-1$ is particularly useful for our purposes here. Then $t \rightarrow-t$ and, hence, this scaling may be thought of as reversing the direction of time and the corresponding evolution may be thought of as that seen when one goes back in time.

Remark (4) : The density $\rho$ and the expansion rates $\lambda_{t}^{i}$ remain finite if the functions $f^{i}$ and their first derivatives $g_{i}$ are finite. If all the higher derivatives $\frac{d^{n} f\left(m^{i}\right)}{d\left(m^{i}\right)^{n}}$ are finite then all the higher time derivatives $\frac{d^{n} \lambda^{i}}{d t^{n}}$ will also be finite. The evolution will then be non singular.

Remark (5) : In our models, we require that $f(x) \rightarrow x$ as $x \rightarrow 0$. Then, in this limit, the universe will evolve as in Einstein's theory. Furthermore, in the interval $0<x<x_{r}$, let $f$ remain positive and bounded, let all the derivatives of $f$ also remain bounded, and let $f(x) \propto\left(x_{r}-x\right)$ as $x \rightarrow x_{r}$. It then follows from the above Remarks that the universe will evolve as in Einstein's theory when $m^{i} \rightarrow 0$ for all $i$, and as its time reversed version when $m^{i} \rightarrow x_{r}$ for all $i$, and the evolution will remain non singular in between. Note that the properties mentioned above are satisfied by $f(x)=\sin x$ for which $x_{r}=\pi$, and also by the class of functions $f(x ; n)$ given in equation (36) for which $x_{r}=2 x_{*}$. Many such examples may be constructed easily.

Remark (6) : Consider a function $f(x)$ which $\rightarrow x$ as $x \rightarrow 0$. Let $f$ be positive and bounded, and let all its derivatives also be bounded, in the interval $0<x<x_{r}$. Then the universe will evolve as in Einstein's theory when $m^{i} \rightarrow 0$ for all $i$ and the evolution will remain non singular until $m^{i}$ approach $x_{r}$. The nature of the evolution in the limit $m^{i} \rightarrow x_{r}$ will depend on the behaviour of $f(x)$ as $x \rightarrow x_{r}$.

In LQC, the function $f(x)=\sin x$ is determined by the underlying theory. In the present LQC - inspired models, the generalisation is empirical and no underlying theory is invoked which may determine $f(x)$. This feature is a shortcoming of the present models. But it may be taken as a strength 
also. One may then consider possible asymptotic behaviours of $f(x)$ from a completely general point of view, obtain a variety of asymptotics of the evolution, and thereby gain insights into the various types of singular and non singular evolutions possible. From such a perspective, it is quite natural to consider the case where $f(x) \propto\left(x_{r}-x\right)^{q}$ in the limit $x \rightarrow x_{r}$. Note that it is also possible that $x_{r}$ is infinite. One may then also consider the cases where, in the limit $x \rightarrow \infty$, the function $f(x) \propto x^{q}$, or $f(x) \rightarrow($ const $)$, or $f(x) \propto e^{-b x}$.

Remark (7) : Consider the isotropic case. Let the equation of state be given by $p=w \rho$ where $w$ is a constant and $1+w>0$. Then, equations (9) and (31) - (33) can be solved explicitly if certain integrations and functional inversions can be performed. Denoting the initial values with 0 -subscripts, it follows from equations (9) and (31) that

$$
\frac{\rho}{\rho_{0}}=\frac{f^{2}}{f_{0}^{2}}=\left(\frac{a}{a_{0}}\right)^{-(1+w) d} \quad, \quad \rho_{0}=\rho_{q m} f_{0}^{2},
$$

which gives $a$ in terms of $m$. Note that if $f$ has a maximum $f_{m x}$ then the density $\rho$ has a maximum $\rho_{m x}$ and the scale factor $a$ has a minimum $a_{m n}$ which are given by

$$
\rho_{m x}=\rho_{q m} f_{m x}^{2}, \quad a_{m n}=a_{0}\left(\frac{f_{0}}{f_{m x}}\right)^{\frac{2}{(1+w) d}} .
$$

Equations (31) and (32) give

$$
\mathcal{F}=-\int \frac{d m}{f^{2}}=c_{q m} \tilde{t}, \quad \tilde{t}=t-t_{0}+\frac{\mathcal{F}_{0}}{c_{q m}}
$$

where $c_{q m}=\frac{(1+w) d}{2 \gamma \lambda_{q m}}$. Equation (39) defines $\mathcal{F}(m)$ and gives $t$ in terms of $m$. Inverting $t(m)$ then gives $m(t)$ which, in turn, gives $a(t)$.

Remark (8) : Consider the isotropic case with $p=w \rho$ as above. Now, let these $p$ and $\rho$ be due to a minimally coupled scalar field $\sigma$ with a potential $V(\sigma)$, see equation (12). Writing $\sigma_{t}^{2}=(1+w) \rho$, equations (31) and (32) give

$$
\sigma_{t}=\left(\sqrt{(1+w) \rho_{q m}}\right) f \quad, \quad \frac{d m}{d \sigma}=\frac{m_{t}}{\sigma_{t}}=-\frac{\gamma \lambda_{q m} \kappa^{2}}{d-1} \sigma_{t}
$$


It then follows that

$$
\mathcal{S}=-\int \frac{d m}{f}=c_{w} \tilde{\sigma} \quad, \quad \tilde{\sigma}=\sigma-\sigma_{0}+\frac{\mathcal{S}_{0}}{c_{w}}
$$

where $c_{w}=\sqrt{\frac{(1+w) \kappa^{2} d}{2(d-1)}}$. Equation $(40)$ defines $\mathcal{S}(m)$ and gives $\sigma$ in terms of $m$, hence in terms of $t$ if $m(t)$ is known. Inverting $\sigma(m)$ gives $m(\sigma)$, and the scalar field potential $V(\sigma)$ then follows from

$$
2 V=(1-w) \rho_{q m} f^{2}
$$

Note that $V$ given in equation (14) follows by taking $f(m)=m$ here, or by applying the above manipulations to equations (10) and (11).

Remark (9) : Consider the isotropic case and consider the evolution near a maximum of the function $f(m)$. Let $m_{b}$ be a maximum of $f$ and, near its maximum, let

$$
f(m) \simeq f_{m x}\left(1-f_{1}\left(m_{b}-m\right)^{2 n}\right)
$$

where $f_{m x}$ and $f_{1}$ are positive constants and, as in equation (36), $n$ is a positive integer which indicates the flatness of $f$ near its maximum. Let $t_{b}$ be the time when $f$ reaches its maximum. Then, as $t \rightarrow t_{b}$, it follows from equations (39), (37), and (40) that

$$
\begin{aligned}
m_{b}-m & \simeq f_{m x}^{2} c_{q m}\left(t-t_{b}\right) \\
a & \simeq a_{m n}\left(1+a_{1}\left(t-t_{b}\right)^{2 n}\right) \\
c_{w}\left(\sigma-\sigma_{b}\right) & \simeq f_{m x} c_{q m}\left(t-t_{b}\right)
\end{aligned}
$$

where $\sigma_{b}$ is a constant, $a_{m n}=a_{0}\left(\frac{f_{0}}{f_{m x}}\right)^{\frac{2}{(1+w) d}}$, and $a_{1}=\frac{2 f_{1}}{(1+w) d}\left(f_{m x}^{2} c_{q m}\right)^{2 n}$. 


\section{Isotropic evolutions}

Consider the isotropic case, with the equation of state given by $p=w \rho$ where $w$ is a constant and $1+w>0$. We will assume that these $p$ and $\rho$ are due to a minimally coupled scalar field $\sigma$ with a potential $V(\sigma)$. We will first study the examples of functions $f(m)$ for which equations $(31)-$ (33) can be solved explicitly. Using these examples, we will then study the asymptotic evolutions for the functions $f(m)$ and the limits given in Remark (6). As clear from Remarks (7) and (8), obtaining explicit solutions depends on whether the integrations in equations (39) and (40), and the consequent functional inversions, can be performed explicitly.

\section{Example I : $\mathbf{f}(\mathbf{m})=\sin \mathbf{m}$}

It turns out that all the required integrations and functional inversions can be performed for the function $f(m)=\sin m$. As can be checked easily, $\mathcal{F}$ and $\mathcal{S}$ are given by

$$
\mathcal{F}=\cot m \quad, \quad e^{\mathcal{S}}=\cot \frac{m}{2} .
$$

Also, $f$ and $\mathcal{S}$ may be expressed in terms of $\mathcal{F}$ :

$$
f=\frac{1}{\sqrt{1+\mathcal{F}^{2}}} \quad, \quad \operatorname{Cosh} \mathcal{S}=\frac{1}{\sin m}=\sqrt{1+\mathcal{F}^{2}} .
$$

Equations (37), (39), and (40) now give the solutions

$$
\left(\frac{a}{a_{0}}\right)^{(1+w) d}=\frac{1+c_{q m}^{2} \tilde{t}^{2}}{1+c_{q m}^{2} \tilde{t}_{0}^{2}} \quad, \quad \operatorname{Cosh}\left(c_{w} \tilde{\sigma}\right)=\sqrt{1+c_{q m}^{2} \tilde{t}^{2}} .
$$

The potential $V(\sigma)$ for the scalar field given in equation (41) now becomes

$$
V(\sigma)=\frac{(1-w) \rho_{q m}}{2 \operatorname{Cosh}^{2}\left(c_{w} \tilde{\sigma}\right)} .
$$

We note, in passing, that the above expressions are the $(d+1)$ dimensional generalisation of the $(3+1)$ dimensional LQC solution given in [18]. 


\section{Example II : $\mathbf{f}(\mathbf{m})=\mathbf{m}^{\mathbf{q}} \quad, \quad \mathbf{m} \geq \mathbf{0}$}

The function $f(m)=m^{q}$ with $m \geq 0$, by itself, may not be of much interest since $f$ has no finite maximum and, generically, the evolution will be singular. However, for this example, all the integrations in equations (39) and (40), and the consequent functional inversions, can be performed which will lead to explicit solutions. Moreover, together with the shifting and the scaling of $m$ described in Remarks (2), (3), and (5), the solutions for this example can be used to understand the asymptotics of the nonsingular evolutions in a variety of cases which may be of interest. With this purpose in mind, we consider this example and present the explicit isotropic solutions. It also turns out that the solutions for the $0<2 q<1$ case exhibit an interesting feature, and the solutions for the $q=\frac{1}{2}$ case are quite intriguing.

Before proceeding with the isotropic case, we first note that when the function $f(x)=x^{q}$, we have $f^{i}=\left(m^{i}\right)^{q}, m^{i} g_{i}=q f^{i}$, and hence

$$
\sum_{j} m^{j} X_{j}=q \sum_{j k} G_{j k} f^{j} f^{k}
$$

Equation (25) then gets simplified and becomes

$$
\left(m^{i}\right)_{t}+\Lambda_{t} m^{i}=\gamma \lambda_{q m} \kappa^{2} \sum_{j} G^{i j}\left\{(2 q-1) \rho-p_{j}\right\}
$$

If $p_{i}=p=w \rho$ then the above equation becomes

$$
\left(m^{i}\right)_{t}+\Lambda_{t} m^{i}=\frac{\gamma \lambda_{q m} \kappa^{2}}{d-1}(2 q-1-w) \rho .
$$

Now consider the completely isotropic case given by equation (30), and the corresponding equations of motion (9) and (31) - (33). Note that the function $g=q m^{q-1}$ now. Hence, equations (31) and (33) give

$$
H^{2}=\frac{q^{2}}{\gamma^{2} \lambda_{q m}^{2}}\left(\frac{\rho}{\rho_{q m}}\right)^{\frac{2 q-1}{q}} .
$$

Note also that for any quantity $Z(m)$ which varies as $m^{\alpha}$, it follows from equations (31) and (32), and from $f=m^{q}$, that

$$
Z_{t}=Z_{m} m_{t} \sim m^{2 q-1+\alpha} \longrightarrow \frac{d^{n} Z}{d t^{n}} \sim m^{n(2 q-1)+\alpha}
$$


If $Z=(\ln a)$ then $\alpha=0$ formally and $\frac{d^{n}(\ln a)}{d t^{n}} \sim m^{n(2 q-1)}$. In the following, we will take the evolution to be singular if any of the time derivatives of ( $\ln a)$ diverges, and to be non singular otherwise.

The general explicit solution for $f=m^{q}$ follows upon performing the integrations in equations (39) and (40), and the consequent functional inversions. We will now present these solutions.

$$
\mathbf{q} \neq \frac{1}{2}
$$

For $q \neq \frac{1}{2}$ or 1 , equations (39) and (40) give

$$
\begin{aligned}
& m^{1-2 q}=(2 q-1) c_{q m}\left(t-t_{0}\right)+m_{0}^{1-2 q} \equiv T \\
& m^{1-q}=(q-1) c_{w}\left(\sigma-\sigma_{0}\right)+m_{0}^{1-q} \equiv A \sigma+B
\end{aligned}
$$

where the constants $A$ and $B$ can be read off easily. It then follows from equation (37) that the scale factor $a$ is given by

$$
\frac{a}{a_{0}}=\left(\frac{m}{m_{0}}\right)^{-\frac{2 q}{(1+w) d}}=\left(\frac{T}{T_{0}}\right)^{\frac{2 q}{(2 q-1)(1+w) d}}
$$

where $T_{0}=m_{0}^{1-2 q}$. And, it follows from equation (41) that the scalar field potential $V$ is given by

$$
2 V(\sigma)=(1-w) \rho_{q m} m^{2 q}=(1-w) \rho_{q m}(A \sigma+B)^{\frac{2 q}{1-q}} .
$$

For $q=1$, one obtains the standard Einstein's equations as described in Remark (1). Thus, for example, one obtains from equations (37) - (40) that

$$
m=\frac{1}{c_{q m} \tilde{t}}=e^{-c_{w} \tilde{\sigma}}, \quad a \sim m^{-\frac{2}{(1+w) d}}=\left(c_{q m} \tilde{t}\right)^{\frac{2}{(1+w) d}} .
$$

The scalar field potential is given by $V \sim m^{2} \sim e^{-2 c_{w} \tilde{\sigma}}$, see equation (14).

The evolution of the universe follows from equations (54), (52), and (51).

$\mathbf{2} \mathbf{q}>\mathbf{1}$ : In this case, as $m \rightarrow 0$, the scale factor $a \rightarrow \infty$, the time $t \rightarrow \infty$, and the time derivatives of $(\ln a)$ will not diverge. The evolution is 
non singular in this limit, and proceeds smoothly as $m$ increases further. As $m \rightarrow \infty$, the scale factor $a \rightarrow 0$ and the time $t \rightarrow t_{s}$ from above where $t_{s}$ is finite and its value can be read off easily but is not important here. Also, the time derivatives of $(\ln a)$ diverge and, hence, the evolution is singular in this limit. Thus, for the $2 q>1$ case, the universe starts with a zero size and a singularity at a finite time in the past, and expands to infinite size in the infinite future with no further singularities.

$\mathbf{0}<\mathbf{2 q}<\mathbf{1}$ : In this case, as $m \rightarrow 0$, the scale factor $a \rightarrow \infty$ and the time $t \rightarrow t_{s}$ from below where $t_{s}$ is finite and its value can be read off easily but not important here. Also, the time derivatives of $(\ln a)$ diverge and, hence, the evolution is singular in this limit. The evolution proceeds smoothly as $m$ increases further. As $m \rightarrow \infty$, the scale factor $a \rightarrow 0$, the time $t \rightarrow-\infty$, and the time derivatives of $(\ln a)$ will not diverge. The evolution is non singular in this limit. Thus, for the $0<2 q<1$ case, universe approaches a zero size in the infinite past but with no singularity, and expands to infinite size with a singularity at a finite time in the future.

Note the interesting feature that there is a singularity when the universe is increasing to infinite size, and no singularity when it is contracting to zero size. Such a feature is opposite to what one usually comes across in Einstein's theory.

$\mathbf{q}<\mathbf{0}:$ In this case, as $m \rightarrow 0$, the scale factor $a \rightarrow 0$ and the time $t \rightarrow t_{s}$ from below where $t_{s}$ is finite and its value can be read off easily but not important here. Also, the time derivatives of ( $\ln a)$ diverge and, hence, the evolution is singular in this limit. The evolution proceeds smoothly as $m$ increases further. As $m \rightarrow \infty$, the scale factor $a \rightarrow \infty$, the time $t \rightarrow-\infty$, and the time derivatives of $(\ln a)$ will not diverge. The evolution is non singular in this limit. Thus, for the $q<0$ case, the universe is infinite in size in the infinite past, and contracts to zero size and a singularity at a finite time in the future.

$$
\mathbf{q}=\frac{1}{2}
$$

Consider now the $q=\frac{1}{2}$ case. Equations (39) and (37) give

$$
m=m_{0} e^{-c_{q m}\left(t-t_{0}\right)}
$$




$$
\frac{a}{a_{0}}=\left(\frac{m}{m_{0}}\right)^{-\frac{2 q}{(1+w) d}}=e^{\frac{t-t_{0}}{2 \gamma \lambda q m}}
$$

where we have used $c_{q m}=\frac{(1+w) d}{2 \gamma \lambda_{q m}}$ in the last equality. Equations (53) and (55) remain valid, and note that equation (55) gives $V \propto(A \sigma+B)^{2}$.

In this case, as $m \rightarrow 0$, the scale factor $a \rightarrow \infty$ and the time $t \rightarrow \infty$. As $m \rightarrow \infty$, the scale factor $a \rightarrow 0$ and the time $t \rightarrow-\infty$. Also, clearly, the time derivatives of $(\ln a)$ will not diverge and, hence, the evolution is non singular. Thus, for the $q=\frac{1}{2}$ case, the universe starts with a zero size in the infinite past, and expands to infinite size in the infinite future with no singularities.

Note that the evolution in the $q=\frac{1}{2}$ case straddles the border between the singular and non singular evolution : in the limit $m \rightarrow 0$, the evolution is non singular for $2 q>1$ and is singular for $2 q<1$; in the limit $m \rightarrow \infty$, the evolution is singular for $2 q>1$ and is non singular for $2 q<1$; for the border case $2 q=1$, the evolution is non singular in both the asymptotic limits.

Also note that, for $q=\frac{1}{2}$, we have an exponentially growing scale factor with the exponential rate set by the parameter $\lambda_{q m}$ alone, which is related to the quantum of the $(d-1)$ dimensional area as in LQC. The density is given by equation (37),

$$
\rho \sim f^{2} \sim a^{-(1+w) d} \sim e^{-c_{q m} t}
$$

and does not remain constant. Also, there is no restriction on $w$, the equation of state parameter. So, this exponential growth of the scale factor is unlike that which occurs in Einstein's theory due to a positive cosmological constant. See equation (50), now with $2 q=1$, to see how this comes about. These results are intriguing and fascinating but their significance, if any, is not clear to us at present.

\section{Example III : $\mathbf{f}(\mathbf{m})=\mathrm{e}^{\mathbf{m}}$}

We consider the function $f(m)=e^{m}$ for the same reasons as given for Example II : solutions can be obtained explicitly. They may then be used 
to understand the asymptotics of the nonsingular evolutions in other cases which may be of interest. For this example, equations (31) and (33) give

$$
H^{2}=\frac{1}{\gamma^{2} \lambda_{q m}^{2}}\left(\frac{\rho}{\rho_{q m}}\right)^{2} .
$$

Equations (39) and (40) give

$$
\begin{aligned}
e^{-2 m} & =2 c_{q m}\left(t-t_{0}\right)+e^{-2 m_{0}} \equiv T \\
e^{-m} & =c_{w}\left(\sigma-\sigma_{0}\right)+e^{-m_{0}} \equiv A \sigma+B
\end{aligned}
$$

where the constants $A$ and $B$ can be read off easily. It then follows from equation (37) that the scale factor $a$ is given by

$$
\frac{a}{a_{0}}=e^{-\frac{2\left(m-m_{0}\right)}{(1+w) d}}=\left(\frac{T}{T_{0}}\right)^{\frac{1}{(1+w) d}}
$$

where $T_{0}=e^{-2 m_{0}}$. And, it follows from equation (41) that the scalar field potential $V$ is given by

$$
2 V(\sigma)=(1-w) \rho_{q m} e^{2 m}=\frac{(1-w) \rho_{q m}}{(A \sigma+B)^{2}} .
$$

Also, for any quantity $Z(m)$ which varies as $m^{\alpha}$, it follows from equations (31) and (32), and from $f=e^{m}$, that

$$
\frac{d^{n} Z}{d t^{n}} \sim\left(\sum_{k=1}^{n} A_{k} m^{\alpha-k}\right) e^{2 n m}
$$

where $A_{k}$ are some constants. The evolution of the universe follows from equations (61), (59), and (63).

As $m \rightarrow-\infty$, the scale factor $a \rightarrow \infty$, the time $t \rightarrow \infty$, and the time derivatives of $(\ln a)$ will not diverge. The evolution is non singular in this limit, and proceeds smoothly as $m$ increases further. As $m \rightarrow \infty$, the scale factor $a \rightarrow 0$ and the time $t \rightarrow t_{s}$ from above where $t_{s}$ is finite and its value can be read off easily but not important here. Also, the time derivatives of ( $\ln a$ ) diverge and, hence, the evolution is singular in this limit. Thus, the 
universe starts with a zero size and a singularity at a finite time in the past, and expands to infinite size in the infinite future with no further singularities.

In Table I below, we tabulate the asymptotic behaviour of the scale factor $a(t)$ in Examples I - III, described above in detail. In the Table, we give the forms of the function $f(m)$ and the asymptotic values of $a(t)$ in the limit $t \rightarrow \pm \infty$ or $t_{s}$ where $t_{s}$ is finite. Also, we use the letter $S$ or $N S$ to denote whether the evolution in this limit is singular or non singular.

\begin{tabular}{|c|c|c|c|c|c|}
\hline & $f(m)$ & & $t \rightarrow-\infty$ & $t \rightarrow t_{s}$ & $t \rightarrow \infty$ \\
\hline I & $\sin m$ & & $\infty, N S$ & $\rightarrow a_{\min } \rightarrow$ & $\infty, N S$ \\
\hline \multirow{4}{*}{ II } & \multirow{4}{*}{$m^{q}$} & $2 q>1$ & & $0, S$ & $\infty, N S$ \\
\hline & & $2 q=1$ & $0, N S$ & $\longrightarrow$ & $\infty, N S$ \\
\hline & & $0<2 q<1$ & $0, N S$ & $\infty, S$ & \\
\hline & & $q<0$ & $\infty, N S$ & $0, S$ & \\
\hline III & $e^{m}$ & & & $0, S$ & $\infty, N S$ \\
\hline
\end{tabular}

Table I : Asymptotic behaviours of the scale factor a $(\mathbf{t})$. Tabulated here are the forms of the function $f(m)$ in Examples I-III, and the asymptotic values of the scale factor $a(t)$ in the limit $t \rightarrow \pm \infty$ or $t_{s}$ where $t_{s}$ is finite. The accompanying letter $S$ or NS denotes whether the 
evolution in this limit is singular or non singular. The entire evolution is non singular only in Example I, where it has a bounce, and in Example II with $2 q=1$, where it has no bounce. The values of $m$ in these limits follow straightforwardly and, hence, are not tabulated here.

\section{Other Examples}

Using Examples II and III, we now study the asymptotic evolutions for the functions $f(m)$ and the limits given in Remark (6). For these functions, we assume that $f(m) \rightarrow m$ in the limit $m \rightarrow 0$ and also that, until $m$ approaches the limit of interest, $f$ remains positive and bounded, and all its derivatives also remain bounded. It then follows that, in the limit $m \rightarrow 0$, the evolution is as in Einstein's theory, the scale factor $a \rightarrow \infty$, the time $t \rightarrow \infty$, and the time derivatives of $(\ln a)$ will not diverge. As $m$ increases from 0 , the time $t$ decreases from $\infty$, the scale factor $a$ decreases from $\infty$ but remains non zero since $f$ remains bounded from above, and the evolution proceeds smoothly with no singularity until $m$ approaches the limit of interest.

We characterise the evolution in these limits of interest as follows. If the time derivatives of $(\ln a)$ do not diverge then the evolution will be referred to as non singular; if the scale factor $a \rightarrow \infty$ then the evolution will be said to have a bounce; when there is a bounce, if the evolution as $a \rightarrow \infty$ is same as that in Einstein's theory then the evolution will be referred to as symmetric; and, when there is a bounce, if the evolution as $a \rightarrow \infty$ is different from that in Einstein's theory then evolution will be referred to as asymmetric.

Note that the words symmetric and asymmetric refer not to the actual shape of $a(t)$, but refer only to whether or not $a(t)$ evolves as in Einstein's theory at both the ends of a bounce where $a \rightarrow \infty$. Thus, for example, if $f(m)=\sin m$, or if $f(m)=f(m ; n)$ given in equation (36), then the evolution is non singular, has a bounce, and is symmetric. It turns out that, because of the symmetric shapes of $\sin m$ and $f(m ; n)$, the shape of $a(t)$ is also symmetric but, generically, this need not be the case. 


\section{Example IV : $\mathbf{f}(\mathbf{m}) \propto\left(\mathbf{m}_{\mathbf{s}}-\mathbf{m}\right)^{\mathbf{q}}$ as $\mathbf{m} \rightarrow \mathbf{m}_{\mathbf{s}}$}

We now consider the Example where the function $f(m) \propto\left(m_{s}-m\right)^{q}$ in the limit of interest $m \rightarrow m_{s}$. The evolution in the limit $m \rightarrow m_{s}$ can be read off from the asymptotic behaviour in Example II as $m \rightarrow 0$ there. One also needs to change the sign of $t$ there since $f \propto\left(m_{s}-m\right)^{q}$ now. It then follows that if $2 q \geq 1$ then, as $m \rightarrow m_{s}$, the scale factor $a \rightarrow \infty$, the time $t \rightarrow-\infty$, and the time derivatives of $(\ln a)$ will not diverge. Hence, the evolution is non singular and is different from that in Einstein's theory unless $q=1$. Thus, as $m$ increases from 0 to $m_{s}$, the universe evolves as in Einstein's theory in the limit $t \rightarrow \infty$, the scale factor $a$ remains non zero throughout, increases to $\infty$ and, unless $q=1$, evolves asymmetrically in the limit $t \rightarrow-\infty$. The evolution has a bounce, is asymmetric unless $q=1$, and remains non singular throughout.

If $0<2 q<1$ then, as $m \rightarrow m_{s}$, the scale factor $a \rightarrow \infty$, the time $t \rightarrow t_{s}$ which is finite, the time derivatives of $(\ln a)$ diverge and, hence, the evolution is singular. Thus, as $m$ increases from 0 to $m_{s}$, the universe evolves as in Einstein's theory in the limit $t \rightarrow \infty$, the scale factor $a$ remains non zero throughout and increases to $\infty$ at a finite time $t_{s}$ in the past. The evolution has a bounce, and is singular as $t \rightarrow t_{s}$.

If $q<0$ then, as $m \rightarrow m_{s}$, the scale factor $a \rightarrow 0$, the time $t \rightarrow t_{s}$ which is finite, the time derivatives of $(\ln a)$ diverge and, hence, the evolution is singular. Thus, as $m$ increases from 0 to $m_{s}$, the universe evolves as in Einstein's theory in the limit $t \rightarrow \infty$, the scale factor $a$ decreases from $\infty$ to 0 at a finite time $t_{s}$ in the past, and the evolution is singular as $t \rightarrow t_{s}$.

\section{Example V: $\mathbf{f}(\mathbf{m}) \propto \mathbf{m}^{\mathbf{q}}$ as $\mathbf{m} \rightarrow \infty$}

We now consider the Example where the function $f(m) \propto m^{q}$ in the limit of interest $m \rightarrow \infty$. The evolution in the limit $m \rightarrow \infty$ can be read off from the asymptotic behaviour in Example II as $m \rightarrow \infty$ there. It then follows that if $2 q>1$ then, as $m \rightarrow \infty$, the scale factor $a \rightarrow 0$, the time $t \rightarrow t_{s}$ which is finite, the time derivatives of $(\ln a)$ diverge and, hence, the evolution is singular. Thus, as $m$ increases from 0 to $\infty$, the universe evolves 
as in Einstein's theory in the limit $t \rightarrow \infty$, the scale factor $a$ decreases from $\infty$ to 0 at a finite time $t_{s}$ in the past, and the evolution is singular as $t \rightarrow t_{s}$.

If $0<2 q \leq 1$ then, as $m \rightarrow \infty$, the scale factor $a \rightarrow 0$, the time $t \rightarrow-\infty$, the time derivatives of $(\ln a)$ will not diverge and, hence, the evolution is non singular. Thus, as $m$ increases from 0 to $\infty$, the universe evolves as in Einstein's theory in the limit $t \rightarrow \infty$, the scale factor $a$ decreases from $\infty$ to 0 as $t \rightarrow-\infty$, and the evolution remains non singular throughout.

If $q<0$ then, as $m \rightarrow \infty$, the scale factor $a \rightarrow \infty$, the time $t \rightarrow-\infty$, the time derivatives of $(\ln a)$ will not diverge and, hence, the evolution is non singular. Thus, as $m$ increases from 0 to $\infty$, the universe evolves as in Einstein's theory in the limit $t \rightarrow \infty$, the scale factor $a$ decreases from $\infty$ to some non zero value and then increases again to $\infty$ as $t \rightarrow-\infty$, the evolution has a bounce, and remains non singular throughout.

\section{Example VI : $\mathbf{f}(\mathbf{m}) \propto \mathbf{1}$ as $\mathbf{m} \rightarrow \infty$}

We now consider the Example where the function $f(m) \propto 1$ in the limit of interest $m \rightarrow \infty$. This Example can be thought of as a special case of Example $\mathrm{V}$ with $q=0$. It follows straightforwardly from equations (37) and (39) that the scale factor $a \rightarrow$ (const) and the time $t \rightarrow-\infty$. Clearly, the time derivatives of $(\ln a)$ will not diverge and, hence, the evolution is non singular. Thus, as $m$ increases from 0 to $\infty$, the universe evolves as in Einstein's theory in the limit $t \rightarrow \infty$, the scale factor $a$ decreases from $\infty$ to some non zero constant value as $t \rightarrow-\infty$, and the evolution remains non singular throughout.

The density $\rho$ also approaches a non zero constant value as $t \rightarrow-\infty$. This phase of the evolution is then similar to what is expected in string/M theory where, as one goes back in time, the ten/eleven dimensional early universe is believed to enter and remain in a Hagedorn phase in which its temperature is of the order of $l_{s}^{-1}$ and its density is of the order of $l_{s}^{-(d+1)}$ where $l_{s}$ is the string length scale [19] - [24]. 


\section{Example VII : $\mathbf{f}(\mathbf{m}) \propto \mathbf{e}^{-\mathbf{b m}}$ as $\mathbf{m} \rightarrow \infty ; \mathbf{b}>\mathbf{0}$}

We now consider the Example where the function $f(m) \propto e^{-b m}$ with $b>$ 0 , in the limit of interest $m \rightarrow \infty$. The evolution in the limit $m \rightarrow \infty$ here can be read off from the asymptotic behaviour in Example III as $m \rightarrow-\infty$ there. One also needs to change the sign of $t$ there since $f \propto e^{-b m}$ now. It then follows that, as $m \rightarrow \infty$, the scale factor $a \rightarrow \infty$, the time $t \rightarrow-\infty$, the time derivatives of $(\ln a)$ will not diverge and, hence, the evolution is non singular. Thus, as $m$ increases from 0 to $\infty$, the universe evolves as in Einstein's theory in the limit $t \rightarrow \infty$, the scale factor $a$ decreases from $\infty$ to some non zero value and then increases again to $\infty$ as $t \rightarrow-\infty$, the evolution has a bounce, and remains non singular throughout.

\section{Summary of Examples IV - VII}

In Table II below, we tabulate the asymptotic behaviour of the scale factor $a(t)$ in Examples IV - VII, described above in detail. In the Table, we give the forms of the function $f(m)$ in the limits of interest and the asymptotic values of $a(t)$ in the limit $t \rightarrow \pm \infty$ or $t_{s}$ where $t_{s}$ is finite. Also, we use the letter $S$ or $N S$ to denote whether the evolution in this limit is singular or non singular.

We now highlight the results of Examples IV - VII by specifically pointing out the cases of non singular evolutions. In all these Examples, by assumption, we have that as $m$ increases from 0 , the universe evolves as in Einstein's theory in the limit $t \rightarrow \infty$, the scale factor $a$ decreases from $\infty$ and remains non zero until $m \rightarrow m_{s}$, or $m \rightarrow \infty$ as the case may be.

- In the Example where $f(m) \propto\left(m_{s}-m\right)^{q}$ as $m \rightarrow m_{s}$, the evolution is non singular and has a bounce if $2 q \geq 1$. It is asymmetric unless $q=1$. Also, $t \rightarrow-\infty$ and $a \rightarrow \infty$ in the limit $m \rightarrow m_{s}$.

- In the Example where $f(m) \propto m^{q}$ as $m \rightarrow \infty$, the evolution is non singular and asymmetric if $2 q \leq 1$. Also, $t \rightarrow-\infty$; and $a \rightarrow 0$ if $0<2 q \leq 1, a \rightarrow$ (const) if $q=0$, and $a \rightarrow \infty$ if $q<0$ in the limit $m \rightarrow \infty$. The evolution for the $q=0$ case in this limit is similar to that expected in the Hagedorn phase of string/M theory. 
- In the Example where $f(m) \propto e^{-b m}$ with $b>0$ as $m \rightarrow \infty$, the evolution is non singular, has a bounce, and is asymmetric. Also, $t \rightarrow$ $-\infty$ and $a \rightarrow \infty$ in the limit $m \rightarrow \infty$.

\begin{tabular}{||c||c|c|c|c|c||}
\hline \hline & $f(m)$ & & $t \rightarrow-\infty$, & $t \rightarrow t_{s}$ & $t \rightarrow \infty$ \\
all NS
\end{tabular}

Table II : Asymptotic behaviours of the scale factor a(t). 
Tabulated here are the forms of the function $f(m)$ in the limits of interest in Examples IV - VII, and the asymptotic values of the scale factor a $(t)$ in

the limit $t \rightarrow \pm \infty$ or $t_{s}$ where $t_{s}$ is finite. In the limit $m \rightarrow 0$ in these Examples, $f(m) \rightarrow m, t \rightarrow \infty, a(t) \rightarrow \infty$ as in Einstein's theory, and the evolution is non singular. The accompanying letter $S$ or $N S$ denotes whether the evolution in this limit is singular or non singular. The entire evolution is non singular in several cases in these Examples.

\section{Anisotropic evolutions}

Consider the anisotropic evolutions. The scale factors $a_{i}=e^{\lambda^{i}}$ are now different for different $i$. Let the equations of state be given by $p_{i}=p=w \rho$ where $w$ is a constant and $1+w>0$. The evolution is described by equations (9) and (24) - (29). In general, we can not solve these equations explicitly for any non trivial function $f(x)$. Therefore, we consider Example II where $f(x)=x^{q}$ and study mainly the asymptotic evolutions in the limit $m^{i} \rightarrow 0$ for all $i$, and in the limit $m^{i} \rightarrow \infty$ for all $i$. Other Examples and other limits may be studied similarly.

When $f(x)=x^{q}$ and $p_{i}=p=w \rho$, we have $f^{i}=f\left(m^{i}\right)=\left(m^{i}\right)^{q}$ and

$$
\rho=\rho_{0} e^{-(1+w)\left(\Lambda-\Lambda_{0}\right)}, \quad X_{j}=q\left(m^{j}\right)^{q-1} \sum_{k} G_{j k}\left(m^{k}\right)^{q}
$$

which follow from equations (9) and (27). Equations (24), (25) or (49), and (26) become

$$
\begin{aligned}
\sum_{i j} G_{i j}\left(m^{i}\right)^{q}\left(m^{j}\right)^{q} & =2 \gamma^{2} \lambda_{q m}^{2} \kappa^{2} \rho_{0} e^{-(1+w)\left(\Lambda-\Lambda_{0}\right)} \\
\left(m^{i}\right)_{t}+\Lambda_{t} m^{i} & =\frac{\gamma \lambda_{q m} \kappa^{2}}{d-1}(2 q-1-w) \rho_{0} e^{-(1+w)\left(\Lambda-\Lambda_{0}\right)} \\
\left(\gamma \lambda_{q m}\right) \lambda_{t}^{i} & =q \sum_{j k} G^{i j} G_{j k}\left(m^{j}\right)^{q-1}\left(m^{k}\right)^{q} .
\end{aligned}
$$

One also has $m^{i}-m^{j}=\mu^{i j} e^{-\Lambda}$ where $\mu^{i j}$ are constants, see equation (29). 


$$
\mathbf{w}=2 \mathbf{q}-1
$$

Explicit solutions can be obtained for the $w=2 q-1$ case. In this case, equation (66) gives

$$
m^{i}=m_{0}^{i} e^{-\left(\Lambda-\Lambda_{0}\right)}
$$

where $m_{0}^{i}$ are constants. Consider equation (65). When $2 q=1+w$, the $e^{\Lambda}$-dependent factors cancel in this equation and one obtains

$$
\sum_{i j} G_{i j}\left(m_{0}^{i}\right)^{q}\left(m_{0}^{j}\right)^{q}=2 \gamma^{2} \lambda_{q m}^{2} \kappa^{2} \rho_{0} .
$$

Consider equation (67). Using equation (68), one obtains

$$
\lambda_{t}^{i}=\lambda_{t 0}^{i} e^{-(2 q-1)\left(\Lambda-\Lambda_{0}\right)}, \quad \Lambda_{t}=\Lambda_{t 0} e^{-(2 q-1)\left(\Lambda-\Lambda_{0}\right)}
$$

where $\lambda_{t 0}^{i}$ and $\Lambda_{t 0}$ are given by

$$
\left(\gamma \lambda_{q m}\right) \lambda_{t 0}^{i}=q \sum_{j k} G^{i j} G_{j k}\left(m_{0}^{j}\right)^{q-1}\left(m_{0}^{k}\right)^{q}, \quad \Lambda_{t 0}=\sum_{i} \lambda_{t 0}^{i} .
$$

Solving the equation for $\Lambda_{t}$ in (70), one obtains

$$
e^{(2 q-1)\left(\Lambda-\Lambda_{0}\right)}=(2 q-1) \Lambda_{t 0} \tilde{t}, \quad \tilde{t}=t-t_{0}+\frac{1}{(2 q-1) \Lambda_{t 0}} .
$$

Then it follows that $\tilde{t}_{0}=\frac{1}{(2 q-1) \Lambda_{t 0}}$

$$
\lambda_{t}^{i}=\frac{\alpha^{i}}{\tilde{t}}, \quad e^{\lambda^{i}-\lambda_{0}^{i}}=\left(\frac{\tilde{t}}{\tilde{t}_{0}}\right)^{\alpha^{i}}, \quad \alpha^{i}=\frac{\lambda_{t 0}^{i}}{(2 q-1) \Lambda_{t 0}},
$$

and that

$$
e^{\Lambda-\Lambda_{0}}=\left(\frac{\tilde{t}}{\tilde{t}_{0}}\right)^{\alpha}, \quad \alpha=\sum_{i} \alpha^{i}=\frac{1}{2 q-1} .
$$

These are the explicit anisotropic solutions for the $w=2 q-1$ case. They are parametrised by the initial values $m_{0}^{i}$ and $\lambda_{0}^{i}$, which then determine the remaining initial values $\rho_{0}, \Lambda_{0}, \lambda_{t 0}^{i}$, and $\Lambda_{t 0}$. The following features of these solutions can now be seen easily.

- Setting $m_{0}^{i}=m_{0}$ for all $i$ in the above expressions gives $\lambda_{t 0}^{i}=\frac{\Lambda_{t 0}}{d}$ and $\alpha^{i}=\frac{1}{(2 q-1) d}$, and leads to the solutions given in Example II with $2 q=1+w$ now; compare equations (54) and (73). 
- When $2 q=1$, hence $w=0$, equation (70) leads to

$$
e^{\lambda^{i}-\lambda_{0}^{i}}=e^{\lambda_{t 0}^{i}\left(t-t_{0}\right)}, \quad e^{\Lambda-\Lambda_{0}}=e^{\Lambda_{t 0}\left(t-t_{0}\right)}
$$

where $\lambda_{t 0}^{i}$ and $\Lambda_{t 0}$ are given by equation (71). Also, if $m_{0}^{i}=m_{0}$ for all $i$ then $\lambda_{t 0}^{i}=\frac{q}{\gamma \lambda_{q m}}$. The solutions given in equation (57) then follow.

- Vacuum solutions follow upon setting $\rho_{0}=0$ in equation (69).

- The above anisotropic solutions for the $w=2 q-1$ case are the analogs of the standard Kasner-type solutions in Einstein's theory. Indeed, for $q=1$, we have $w=1$ and equations (71), (74), (69), and (73) give

$$
\gamma \lambda_{q m} \lambda_{t 0}^{i}=m_{0}^{i}, \quad \sum_{i} \alpha^{i}=1, \quad 1-\sum_{i}\left(\alpha^{i}\right)^{2}=\frac{2 \kappa^{2} \rho_{0}}{\Lambda_{t 0}^{2}}
$$

For the vacuum case, $\rho_{0}=0$ and one obtains $\sum_{i} \alpha^{i}=\sum_{i}\left(\alpha^{i}\right)^{2}=1$.

$$
\mathbf{w} \neq 2 \mathrm{q}-1
$$

When $w \neq 2 q-1$, we can not solve equations (65) - (67) explicitly. Therefore, for these cases, we study only the asymptotic evolutions in the limit $m^{i} \rightarrow 0$ for all $i$, and in the limit $m^{i} \rightarrow \infty$ for all $i$. In the asymptotic limits, depending on the value of $q$ and depending on whether $m^{i} \rightarrow 0$ or $m^{i} \rightarrow \infty$ for all $i$, the time $t \rightarrow \pm \infty$ or, after incorporating a finite shift, $t \rightarrow 0$. Below, for the sake of simplicity, we further restrict our study to only those cases where $2 q \neq 1$ and where $t \rightarrow \infty$ or $t \rightarrow 0$. Other cases can be studied similarly. In these asymptotic limits, let $m^{i}, e^{\lambda^{i}}$, and $\lambda_{t}^{i}$ be given by the ansatz

$$
m^{i}=\frac{c^{i}}{t^{b}}, \quad e^{\lambda^{i}} \propto t^{\alpha^{i}} \longleftrightarrow \lambda_{t}^{i}=\frac{\alpha^{i}}{t}
$$

where $b, c^{i}$, and $\alpha^{i}$ are constants which must be determined consistently by equations $(65)-(67)$. The present ansatz gives

$$
f^{i}=\frac{\left(c^{i}\right)^{q}}{t^{b q}}, \quad X_{j}=\frac{x_{j}}{t^{b(2 q-1)}}, \quad \Lambda_{t}=\frac{\alpha}{t}
$$


where

$$
x_{j}=q\left(c^{j}\right)^{q-1} \sum_{k} G_{j k}\left(c^{k}\right)^{q}, \quad \alpha=\sum_{i} \alpha^{i} .
$$

We then have, since $\rho \propto e^{-(1+w) \Lambda}$,

$$
e^{\Lambda}=c_{\Lambda} t^{\alpha}, \quad \rho=\frac{c_{\rho}}{t^{(1+w) \alpha}}
$$

where $c_{\Lambda}$ and $c_{\rho}$ are constants. Equations (29) and (65) - (67) now give

$$
\begin{aligned}
\frac{c^{i}-c^{j}}{t^{b}} & =\frac{\mu^{i j}}{c_{\Lambda} t^{\alpha}} \\
\frac{\sum_{i j} G_{i j}\left(c^{i}\right)^{q}\left(c^{j}\right)^{q}}{t^{2 b q}} & =\frac{2 \gamma^{2} \lambda_{q m}^{2} \kappa^{2} c_{\rho}}{t^{(1+w) \alpha}} \\
\frac{(\alpha-b) c^{i}}{t^{1+b}} & =\frac{\gamma \lambda_{q m} \kappa^{2}(2 q-1-w) c_{\rho}}{(d-1) t^{(1+w) \alpha}} \\
\left(\gamma \lambda_{q m}\right) \frac{\alpha^{i}}{t} & =\frac{\sum_{j} G^{i j} x_{j}}{t^{b(2 q-1)}} .
\end{aligned}
$$

Equation (82) determines $b$ in terms of $q$ and relates $\alpha^{i}$ and $x^{i}$, equivalently $\alpha^{i}$ and $c^{i}$ :

$$
b=\frac{1}{2 q-1}, \quad\left(\gamma \lambda_{q m}\right) \alpha^{i}=\sum_{j} G^{i j} x_{j} .
$$

We now analyse equations $(79)-(81)$. Note that $1+b=2 b q$.

$$
\left(\frac{1}{\mathrm{t}^{2 b q}}\right) \gg\left(\frac{1}{\mathfrak{t}^{(1+w) \alpha}}\right) \quad \longrightarrow \text { Asymptotic Anisotropy }
$$

Consider first the case where $\left(\frac{1}{t^{2 b q}}\right) \gg\left(\frac{1}{t^{(1+w) \alpha}}\right)$ in the asymptotic limits. Then, since $1+b=2 b q$, the right hand sides of both the equations (80) and (81) can be neglected and, hence, the resulting solution is equivalent to the vacuum solutions obtained earlier. Thus, one now obtains

$$
\alpha-b=\sum_{i j} G_{i j}\left(c^{i}\right)^{q}\left(c^{j}\right)^{q}=0,
$$

see equations (69) with $\rho_{0}=0$ and (74). Since $\alpha-b=0$, equation (81) does not impose any further restriction on $c^{i}$. Also, with $\alpha=b$, equation (79) 
simply determines the constants $\mu^{i j}$ and does not restrict $\left(c^{i}-c^{j}\right)$. This means that $c^{i}$, and hence $m^{i}$ and $\lambda_{t}^{i}$, are generically different for different $i$ and, therefore, the evolution is anisotropic. The volume factor $e^{\Lambda}$ is now given by

$$
e^{\Lambda} \propto t^{\alpha} \propto t^{\frac{1}{2 q-1}}
$$

and, depending on the value of $q$, it may vanish or diverge asymptotically.

- Note that the condition $\left(\frac{1}{t^{2 b q}}\right) \gg\left(\frac{1}{t^{(1+w) \alpha}}\right)$ and the consequent anisotropic evolution are possible in the asymptotic limit $t \rightarrow 0$ only if $2 b q>(1+w) \alpha$, and possible in the asymptotic limit $t \rightarrow \infty$ only if $2 b q<(1+w) \alpha$. Also, note that $\alpha=b=\frac{1}{2 q-1}$ and we have assumed that $1+w>0$.

- Thus, anisotropic evolution is possible in the limit $t \rightarrow 0$ only if $2 b q>(1+w) b$. If $2 q>1$ then $b>0$ and this inequality implies that $w<2 q-1$. If $0<2 q<1$ then $b<0$ and this inequality implies that $w>2 q-1$.

- Similarly, anisotropic evolution is possible in the limit $t \rightarrow \infty$ only if $2 b q<(1+w) b$. If $2 q>1$ then $b>0$ and this inequality implies that $w>2 q-1$. If $0<2 q<1$ then $b<0$ and this inequality implies that $w<2 q-1$.

- If $q<0$ then $b<0, b q>0$ and, hence, $2 b q>(1+w) \alpha$ always. This means that, when $q<0$, anisotropic evolution is possible only in the asymptotic limit $t \rightarrow 0$.

- Note that equations (84) are the analogs of Kasner's solutions in Einstein's theory. Indeed, for $q=1$, equations (83) give $b=1$ and $\left(\gamma \lambda_{q m}\right) \alpha^{i}=c^{i}$; and, equation (84) then gives

$$
\alpha-1=0, \quad \alpha^{2}-\sum_{i}\left(\alpha^{i}\right)^{2}=0
$$

Also, since $2 q-1=1$ now, one has the familar result that the evolution is anisotropic in the limit $t \rightarrow 0$ if $w<1$, and it is anisotropic in the limit $t \rightarrow \infty$ if $w>1$. 


$$
\left(\frac{1}{\mathbf{t}^{2 \mathrm{bq}}}\right)=\left(\frac{1}{\mathbf{t}^{(1+\mathrm{w}) \alpha}}\right) \longrightarrow \text { Asymptotic Isotropy }
$$

Consider next the case where $\left(\frac{1}{t^{2 b q}}\right)=\left(\frac{1}{t^{(1+w) \alpha}}\right)$ in the asymptotic limits. Since $1+b=2 b q$, the right hand sides of both the equations (80) and (81) are now comparable. One then obtains $2 b q=(1+w) \alpha$ and

$$
\alpha=\frac{2 q}{(2 q-1)(1+w)}, \quad \alpha-b=\frac{2 q-1-w}{(2 q-1)(1+w)} .
$$

Note that this value of $\alpha$ follows in the isotropic case also, see equation (54). Equation (80) gives

$$
\sum_{i j} G_{i j}\left(c^{i}\right)^{q}\left(c^{j}\right)^{q}=2 \gamma^{2} \lambda_{q m}^{2} \kappa^{2} c_{\rho}
$$

Consider equation (81). If $2 q=1+w$ then $\alpha-b=0$, equation (81) is identically satisfied and, therefore, imposes no restriction on $c^{i}$. Also, since $\alpha=b$ now, equation (79) simply determines the constants $\mu^{i j}$ and does not restrict $\left(c^{i}-c^{j}\right)$. This means that $m^{i}$ are generically different for different $i$ and, hence, the evolution is anisotropic. This case where $2 q=1+w$ has been studied in the earlier part of this section and explicit solutions have also been presented.

Let $2 q-1-w \neq 0$. Then $\alpha-b \neq 0$, these factors now cancel each other in equation (81), and one obtains

$$
c^{i}=\frac{\gamma \lambda_{q m} \kappa^{2}}{d-1}(2 q-1)(1+w) c_{\rho}
$$

Thus $c^{i}$, and hence $m^{i}$ and $\lambda_{t}^{i}$, are same for all $i$. Then $\alpha^{i}=\frac{\alpha}{d}$, see equation (54) also. This means that the evolution is isotropic. Now, this conclusion can be consistent with equation (79) only if $\left(\frac{1}{t^{b}}\right) \gg\left(\frac{1}{t^{\alpha}}\right)$ in the asymptotic limits. Then, the right hand side of equation (79) can be negelcted which then implies that $c^{i}-c^{j}=0$ for all $i$ and $j$ and, hence, that the evolution is isotropic. The volume factor $e^{\Lambda}$ is now given by

$$
e^{\Lambda} \propto t^{\alpha} \propto t^{\frac{2 q}{(2 q-1)(1+w)}}
$$

and, depending on the value of $q$, it may vanish or diverge asymptotically. 
- Note that the condition $\left(\frac{1}{t^{b}}\right) \gg\left(\frac{1}{t^{\alpha}}\right)$ and the consequent isotropic evolution are possible in the asymptotic limit $t \rightarrow 0$ only if $b>\alpha$, and possible in the asymptotic limit $t \rightarrow \infty$ only if $b<\alpha$. Also, note that $b=\frac{1}{2 q-1}$ and $\alpha=\frac{2 b q}{1+w}$ and we have assumed that $1+w>0$.

- Thus, isotropic evolution is possible in the limit $t \rightarrow 0$ only if $b>\frac{2 b q}{1+w}$. If $2 q>1$ then $b>0$ and this inequality implies that $w>2 q-1$. If $0<2 q<1$ then $b<0$ and this inequality implies that $w<2 q-1$.

- Similarly, isotropic evolution is possible in the limit $t \rightarrow \infty$ only if $b<\frac{2 b q}{1+w}$. If $2 q>1$ then $b>0$ and this inequality implies that $w<2 q-1$. If $0<2 q<1$ then $b<0$ and this inequality implies that $w>2 q-1$.

- If $q<0$ then $b<0, \alpha>0$ and, hence, $b<\alpha$ always. This means that, when $q<0$, isotropic evolution is possible only in the asymptotic limit $t \rightarrow \infty$.

\section{Conclusion}

We now present a brief summary and conclude by mentioning several issues for further studies. In this paper, we considered the LQC - inspired models which generalise the effective equations of LQC to $(d+1)$ dimensions and the function $\sin x$ to an arbitrary function $f(x)$, see equations (22) and (23). Then, assuming that $p=w \rho$ and $(1+w)>0$, we studied a variety of $(d+1)$ dimensional cosmological evolutions in these models corresponding to a variety of possible behaviours of the function $f(x)$. We found explicit solutions for the isotropic cases when $f(x)=\sin x, x^{q}$, and $e^{x}$. For these functions, we also found the potential $V(\sigma)$ for a minimally coupled scalar field $\sigma$ which may give rise to the equation of state $p=w \rho$. We found anisotropic Kasner-type solutions when $f(x)=x^{q}$ and $w=2 q-1$.

Together with the shift and the scaling symmetries of the effective equations, we then used the explicit solutions to describe the asymptotic evolutions in other examples of $f(x)$ : examples where $f(x) \rightarrow x$ in the limit $x \rightarrow 0$ and $f(x) \propto\left(x_{r}-x\right)^{q}$ in the limit $x \rightarrow x_{r}$ or, in the limit $x \rightarrow \infty$, the 
function $f(x) \propto x^{q}$, or $f(x) \rightarrow$ (const), or $f(x) \propto e^{-b x}$. Such asymptotic behaviours are quite natural and, therefore, they may apply to a wide class of functions $f(x)$.

We find that, depending on $f(x)$ in the LQC - inspired models, a variety of cosmological evolutions are possible, singular as well as non singular. Even in the cases where there is a bounce and no singularities, the asymptotic evolutions are generically different from that in Einstein's theory. We also found an intriguing and fascinating result that, for $f(x)=\sqrt{x}$, the evolution is non singular and $a(t)$ grows exponentially at a rate set by $\lambda_{q m}$, the quantum parameter related to the area quantum. But its significance, if any, is not clear to us at present.

We now conclude by mentioning several issues for further studies. It is worthwhile to understand whether, how, and in what fundamental theories, the effective equations of the LQC - inspired models and, in particular, the function $f(x)$ may arise. One may also study whether similar effective equations can be constructed, even if only empirically, and applied to black hole singularities. It will indeed be interesting if black hole singularities may also be resolved in a variety of ways depending on some empirical function(s) in such models. The present LQC - inspired models may also be applied in the context of $\mathrm{M}$ theory cosmology where, due to $\mathrm{U}$ duality symmetries and appropriate intersecting brane configurations, $(10+1)$ dimensional early universe evolves to a $(3+1)$ dimensional universe, with the remaining seven directions remaining constant in size [24, 26, 27, 28].

Note that one way to adapt our LQC-inspired model to study black hole singularities would be to construct the covarint version of the effective equations (24) - (26) which would generalise Einstien's equations (2). For various proposals for covariantising the effective equations in LQC, see [29] [34]. Such a covariant formulation is likely to have other applications too. For example, it may be used to study the evolutions of cosmological perturbations across a bounce.

Making progress on any of these issues seems difficult but it also seems worthwhile to pursue them.

Acknowledgement: We thank G. Date for helpful comments. 


\section{References}

[1] M. Bojowald, Absence of singularity in loop quantum cosmology, Phys. Rev. Lett. 86, 5227 (2001), doi:10.1103/PhysRevLett.86.5227, [gr-qc/0102069];

The Inverse scale factor in isotropic quantum geometry, Phys. Rev. D 64, 084018 (2001), doi:10.1103/PhysRevD.64.084018, [gr-qc/0105067];

Isotropic loop quantum cosmology, Class. Quant. Grav. 19, 2717 (2002), doi:10.1088/0264-9381/19/10/313, [gr-qc/0202077];

Homogeneous loop quantum cosmology, Class. Quant. Grav. 20, 2595 (2003), doi:10.1088/0264-9381/20/13/310, [gr-qc/0303073].

[2] A. Ashtekar, M. Bojowald and J. Lewandowski, Mathematical structure of loop quantum cosmology, Adv. Theor. Math. Phys. 7, 233 (2003), doi:10.4310/ATMP.2003.v7.n2.a2, [gr-qc/0304074].

[3] A. Ashtekar, T. Pawlowski and P. Singh, Quantum nature of the big bang, Phys. Rev. Lett. 96, 141301 (2006), doi:10.1103/PhysRevLett.96.141301, [gr-qc/0602086];

Quantum Nature of the Big Bang: Improved dynamics, Phys. Rev. D 74, 084003 (2006), doi:10.1103/PhysRevD.74.084003, [gr-qc/0607039].

[4] A. Ashtekar and P. Singh, Loop Quantum Cosmology: A Status Report, Class. Quant. Grav. 28, 213001 (2011), doi:10.1088/0264-9381/28/21/213001, [arXiv:1108.0893 [gr-qc]].

[5] G. Date, Lectures on $L Q G / L Q C$, arXiv:1004.2952 [gr-qc].

[6] A. Ashtekar, New Variables for Classical and Quantum Gravity, Phys. Rev. Lett. 57, 2244 (1986), doi:10.1103/PhysRevLett.57.2244; New Hamiltonian Formulation of General Relativity, Phys. Rev. D 36, 1587 (1987), doi:10.1103/PhysRevD.36.1587; A. Ashtekar and J. Lewandowski, Background independent quantum gravity: A Status report, 
Class. Quant. Grav. 21, R53 (2004),

doi:10.1088/0264-9381/21/15/R01, [gr-qc/0404018].

[7] A. Ashtekar, Lectures on non-perturbative canonical gravity, Notes prepared in collaboration with R. S. Tate, World Scientific, Singapore (1991);

C. Rovelli, Quantum Gravity, Cambridge University Press, Cambridge (2004);

T. Thiemann, Introduction to modern canonical quantum general relativity, Cambridge University Press, Cambridge (2005);

C. Rovelli and F. Vidotto, Covariant loop quantum gravity, Cambridge University Press, Cambridge (2014).

[8] G. Date, Absence of the Kasner singularity in the effective dynamics from loop quantum cosmology, Phys. Rev. D 71, 127502 (2005), doi:10.1103/PhysRevD.71.127502, [gr-qc/0505002].

[9] A. Ashtekar and E. Wilson-Ewing, Loop quantum cosmology of Bianchi I models, Phys. Rev. D 79, 083535 (2009), doi:10.1103/PhysRevD.79.083535, [arXiv:0903.3397 [gr-qc]].

[10] L. Linsefors and A. Barrau, Modified Friedmann equation and survey of solutions in effective Bianchi-I loop quantum cosmology, Class. Quant. Grav. 31, 015018 (2014), doi:10.1088/0264-9381/31/1/015018, [arXiv:1305.4516 [gr-qc]].

[11] P. Diener, A. Joe, M. Megevand and P. Singh, Numerical simulations of loop quantum Bianchi-I spacetimes, Class. Quant. Grav. 34, 094004 (2017), doi:10.1088/1361-6382/aa68b5, [arXiv:1701.05824 [gr-qc]].

[12] S. Kalyana Rama, A Class of LQC-inspired Models for Homogeneous, Anisotropic Cosmology in Higher Dimensional Early Universe, Gen. Rel. Grav. 48, 155 (2016), doi:10.1007/s10714-016-2150-2, arXiv:1608.03231 [gr-qc].

[13] N. Bodendorfer, T. Thiemann and A. Thurn, New Variables for Classical and Quantum Gravity in all Dimensions I. Hamiltonian Analysis, 
Class. Quant. Grav. 30, 045001 (2013),

doi:10.1088/0264-9381/30/4/045001, [arXiv:1105.3703 [gr-qc]].

[14] N. Bodendorfer, T. Thiemann and A. Thurn, New Variables for Classical and Quantum Gravity in all Dimensions II. Lagrangian Analysis, Class. Quant. Grav. 30, 045002 (2013),

doi:10.1088/0264-9381/30/4/045002, [arXiv:1105.3704 [gr-qc]].

[15] N. Bodendorfer, T. Thiemann and A. Thurn, New Variables for Classical and Quantum Gravity in all Dimensions III. Quantum Theory, Class. Quant. Grav. 30, 045003 (2013),

doi:10.1088/0264-9381/30/4/045003, [arXiv:1105.3705 [gr-qc]].

[16] S. Kalyana Rama and Arnab Priya Saha, Unpublished notes.

[17] X. Zhang, Higher dimensional Loop Quantum Cosmology, Eur. Phys. J. C 76, no. 7, 395 (2016), doi:10.1140/epjc/s10052-016-4249-8, [arXiv:1506.05597 [gr-qc]].

[18] J. Mielczarek, Multi-fluid potential in the loop cosmology, Phys. Lett. B 675, 273 (2009), doi:10.1016/j.physletb.2009.04.034, arXiv:0809.2469 [gr-qc];

E. Wilson-Ewing, The Matter Bounce Scenario in Loop Quantum Cosmology, JCAP 03, 026 (2013), doi:10.1088/1475-7516/2013/03/026, arXiv:1211.6269 [gr-qc].

[19] M. J. Bowick and L. C. R. Wijewardhana, Superstrings at High Temperature, Phys. Rev. Lett. 54, 2485 (1985), doi:10.1103/PhysRevLett.54.2485;

Superstring Gravity and the Early Universe, Gen. Rel. Grav. 18, 59 (1986), doi:10.1007/BF00843749.

[20] R. H. Brandenberger and C. Vafa, Superstrings in the Early Universe, Nucl. Phys. B 316, 391 (1989), doi:10.1016/0550-3213(89)90037-0.

[21] A. A. Tseytlin and C. Vafa, Elements of string cosmology, Nucl. Phys. B 372, 443 (1992), doi:10.1016/0550-3213(92)90327-8, [hep-th/9109048] 
[22] G. Veneziano, A Model for the big bounce, JCAP 03, 004 (2004), doi:10.1088/1475-7516/2004/03/004, [hep-th/0312182].

[23] A. Nayeri, R. H. Brandenberger and C. Vafa, Producing a scale-invariant spectrum of perturbations in a Hagedorn phase of string cosmology, Phys. Rev. Lett. 97, 021302 (2006), doi:10.1103/PhysRevLett.97.021302, [hep-th/0511140].

[24] S. Kalyana Rama, A Stringy correspondence principle in cosmology, Phys. Lett. B 638, 100 (2006), doi:10.1016/j.physletb.2006.05.047, [hep-th/0603216];

A Principle to Determine the Number $(3+1)$ of Large Spacetime Dimensions, Phys. Lett. B 645, 365 (2007), doi:10.1016/j.physletb.2006.11.077, [hep-th/0610071].

[25] N. Bodendorfer, Black hole entropy from loop quantum gravity in higher dimensions, Phys. Lett. B 726, 887 (2013), doi:10.1016/j.physletb.2013.09.043, [arXiv:1307.5029 [gr-qc]].

[26] B. D. Chowdhury and S. D. Mathur, Fractional Brane State in the Early Universe, Class. Quant. Grav. 24, 2689 (2007), doi:10.1088/0264-9381/24/10/014, [hep-th/0611330].

[27] S. Kalyana Rama, Entropy of anisotropic universe and fractional branes, Gen. Rel. Grav. 39, 1773 (2007), doi:10.1007/s10714-007-0488-1, [hep-th/0702202 [hep-th]];

Consequences of $U$ dualities for Intersecting Branes in the Universe, Phys. Lett. B 656, 226 (2007), doi:10.1016/j.physletb.2007.09.069, [arXiv:0707.1421 [hep-th]].

[28] S. Bhowmick and S. Kalyana Rama, $10+1$ to $3+1$ in an Early Universe with mutually BPS Intersecting Branes, Phys. Rev. D 82, 083526 (2010), doi:10.1103/PhysRevD.82.083526, [arXiv:1007.0205 [hep-th]].

[29] G. J. Olmo and P. Singh, Effective Action for Loop Quantum Cosmology a la Palatini, JCAP 01, 030 (2009), doi:10.1088/1475-7516/2009/01/030, arXiv:0806.2783 [gr-qc]. 
[30] T. P. Sotiriou, Covariant Effective Action for Loop Quantum Cosmology from Order Reduction, Phys. Rev. D 79, 044035 (2009), doi:10.1103/PhysRevD.79.044035, [arXiv:0811.1799 [gr-qc]].

[31] G. Date and S. Sengupta, Effective Actions from Loop Quantum Cosmology: Correspondence with Higher Curvature Gravity, Class. Quant. Grav. 26, 105002 (2009), doi:10.1088/0264-9381/26/10/105002, [arXiv:0811.4023 [gr-qc]].

[32] C. Barragan, G. J. Olmo and H. Sanchis-Alepuz, Bouncing Cosmologies in Palatini f(R) Gravity, Phys. Rev. D 80, 024016 (2009), doi:10.1103/PhysRevD.80.024016, arXiv:0907.0318 [gr-qc].

[33] R. C. Helling, Higher curvature counter terms cause the bounce in loop cosmology, arXiv:0912.3011 [gr-qc].

[34] C. Barragan and G. J. Olmo, Isotropic and Anisotropic Bouncing Cosmologies in Palatini Gravity, Phys. Rev. D 82, 084015 (2010), doi:10.1103/PhysRevD.82.084015, arXiv:1005.4136 [gr-qc]. 